\title{
Transition to chip serration in simulated cutting of metallic glasses
}

\author{
Karina E. Avila, Vardan Hoviki Vardanyan, and Herbert M. Urbassek ${ }^{\mathrm{a}}(\mathbb{0}$ \\ Physics Department and Research Center OPTIMAS, University Kaiserslautern, Erwin-Schrödinger-Straße, \\ 67663 Kaiserslautern, Germany
}

Received 26 April 2021 / Accepted 8 July 2021 / Published online 26 July 2021

(C) The Author(s) 2021

\begin{abstract}
Cutting of metallic glasses produces as a rule serrated and segmented chips in experiments, while atomistic simulations produce straight unserrated chips. We demonstrate here that with increasing depth of cut - with all other parameters unchanged - chip serration starts to affect the morphology of the chip also in molecular dynamics simulations. The underlying reason is the shear localization in shear bands. As the distance between shear bands increases with increasing depth of cut, the surface morphology of the chip becomes increasingly segmented. The parallel shear bands that formed during cutting do no longer interact with each other when their separation is $\gtrsim 10 \mathrm{~nm}$. Our results are analogous to the so-called fold instability that has been found when machining nanocrystalline metals.
\end{abstract}

\section{Introduction}

Cutting of materials is a basic machining process whose fundamentals have been studied for long. An established, albeit simplified, model $[1,2]$ assumes that the separation of the material and the formation of the chip can be understood by a simple laminar-flow model, in which the material in front of the tool edge flows towards the tool and separates at the tool edge into a downward component, which flows below the tool, and an upwards component, which forms the chip. Assuming this flow as laminar, the geometry of the tool (in particular the rake angle) determines via the continuity equation the width of the forming chip; in addition, the forces applied on the tool can be calculated [1-5]. As the flow is laminar, the chip geometry is straight (prismatic). Previous atomistic simulations [69] proved that this simple geometry is able to describe in high quantitative accuracy the processes occurring in metallic-glass cutting and chip formation [7]. In experiments, however, as a rule, serrated and segmented chips are found when cutting metallic glasses [10-17].

The physical origin of this discrepancy between an atomistic simulation and an experiment may be expected to lie in the nature of plasticity in metallic glasses. While dislocations are the main agent in crystal plasticity, the plasticity of metallic glasses is governed entirely by shear bands [18-20]. These are regions of shear localization that manifest the intrinsic mechanism that mediates plastic deformation in metallic glasses [18-20]. Considerable effort has been dedicated

\footnotetext{
a e-mail: urbassek@rhrk.uni-kl.de (corresponding author) URL: http://www.physik.uni-kl.de/urbassek/
}

to the understanding of shear-band initiation and propagation [18,21-23]. A shear band is a nanometer-sized plane that undergoes severe shear rearrangements [20] but may reach several hundred micrometers into the amorphous matrix [24-26]. The inhomogeneous nature of strain localization lets one expect that the laminar nature of the flow, which assumes an isotropic and homogeneous response of the material, breaks down. The severe shear rearrangement causes the disruption of the icosahedral network and leads to significant density changes $[27,28]$. Shear bands generated with various deformation mechanisms have been studied both experimentally $[18,21-23,29]$ and in simulations [3037]. For the case of cutting simulations, the formation of a series of equidistant parallel shear bands in straight chips has been reported [6-9]; earlier cutting simulations [38-40] focused on the determination of cutting forces and did not report on the plasticity mechanisms.

In this paper, we study to what extent shear-band formation leads to serrated chips for nanoscopic cutting geometries such as they are studied by molecular dynamics simulation. Using various cutting depths, the geometry, and in particular the spacing of the shear bands formed under cutting is influenced. We shall show that these features affect the chip morphology such that for deep cuts, plastic flow ceases to be laminar and a serrated chip is produced.

\section{Simulation details}

To perform large-scale machining simulations, we create a sufficiently large sample of a $\mathrm{Cu}_{64.5} \mathrm{Zr}_{35.5}$ metal- 


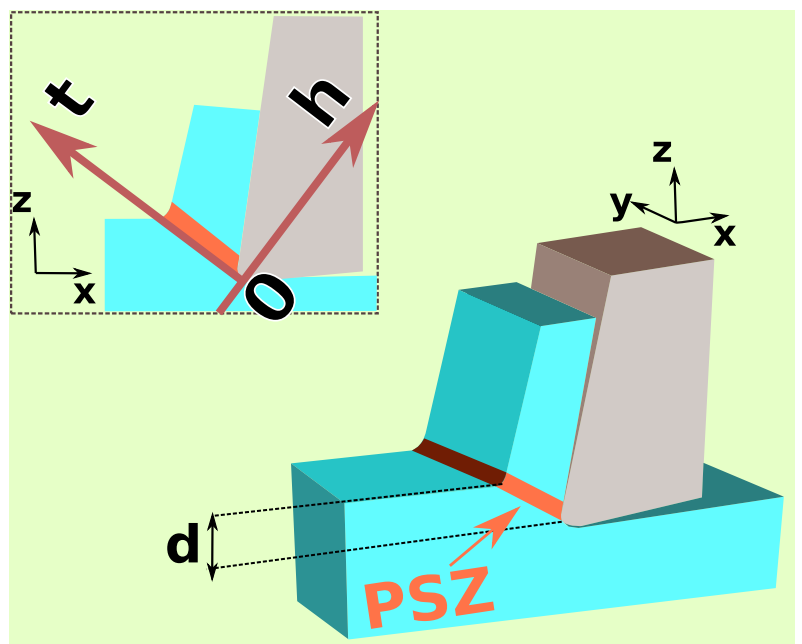

Fig. 1 Schematic setup of the cutting simulations with a depth of cut, $d$. The tool, sample and primary shear zone (PSZ) are displayed in gray, cyan and red, respectively. The inset highlights the coordinates $t$ (parallel to the PSZ) and $h$ (perpendicular to the PSZ) used for the analysis of the shear bands

lic glass containing around 7,903,000 atoms. For our simulations we use the open-source code LAMMPS [41] where the atomic interactions are modeled via an embedded-atom-method potential [42]. The simulation box has dimensions of $101.0 \mathrm{~nm} \times 22.4 \mathrm{~nm} \times 56.1 \mathrm{~nm}$ (see Fig. 1). To prepare the sample, a crystalline mixture is first heated to a temperature $T=2000 \mathrm{~K}$ for a time period of $500 \mathrm{ps}$ and then cooled down to a final temperature of $T=0.1 \mathrm{~K}$ with a quenching rate of $0.01 \mathrm{~K} / \mathrm{ps}$. We demonstrated in earlier work that the plastic properties of this metallic glass provide a realistic description of a $\mathrm{ZrCu}$ glass [33].

The cutting tool is built from $\sim 2,480,000 \mathrm{C}$ atoms that are rigidly arranged. The geometrical shape of the tool has a rounded edge with a curvature radius of 1 $\mathrm{nm}$, a rake angle of $8^{\circ}$ and a clearance angle of $5^{\circ}$ (see Fig. 1). The sample and the cutting tool interact via a purely repulsive Lennard-Jones potential cut-off at the potential minimum. The Lennard-Jones parameters were obtained from Ref. [43]. In this atomistic model, there is no friction between the tool and the workpiece. This is a standard assumption in atomistic models of machining as long as tool wear is not investigated [44]; models implementing friction would require attractive forces between tool and workpiece that need to be based on the chemical interactions between them [45-48].

At the beginning of the simulation, the tool is positioned just outside the interaction range of the LennardJones potential. Then the sample is cut with a cutting speed of $20 \mathrm{~m} / \mathrm{s}$ up to a total cutting length of $l=40$ $\mathrm{nm}$. We use several cutting depths $d$, see Fig. 1, taken as $d=6 \mathrm{~nm}, 12 \mathrm{~nm}, 18 \mathrm{~nm}$, and $24 \mathrm{~nm}$. We fix 10 atomic layers at the bottom and at the left boundary of the sample in order to mimic the immobile bulk of a metallic glass in a real experiment and to avoid centerof-mass translation and rotation of the entire sample.
The shear strain in the vicinity of the fixed layer is monitored throughout the simulation to guarantee that the sample is sufficiently large to avoid any interaction of shear bands with the boundaries. Free boundary conditions are applied to the top and right boundaries, and periodic boundary conditions in the $y$ direction. The temperature is fixed to $T=0.1 \mathrm{~K}$ during the entire cutting simulation. By fixing the temperature at such a low value, thermally activated processes are minimized [33]. Such conditions appear necessary to compensate for the high quenching rates (as compared to experiment) used in the preparation of the sample. However, the disadvantage of this method is that temperature increases within the developing and propagating shear bands that were reported by Cao et al. [28] are not taken into account.

For rendering the atomistic configurations, the open source visualization tool (OVITO) [49] is used.

\section{Results}

\subsection{Plasticity and chip shape}

The upper part of Fig. 2 shows snapshots of the vonMises shear strain (VMSS) at various cutting depths. These snapshots show the final stage of the cutting simulation at cutting length $l=40 \mathrm{~nm}$. One can observe in this figure that parallel shear bands formed along the chip. For the shallowest cut, 8 shear bands can be distinguished, while we see only 4 shear bands for the deeper cuts. In addition, a clear chip serration is seen for cutting depths $\geq 12 \mathrm{~nm}$. Chip serration is commonly observed in experimental studies [10-17]. However, to our knowledge, it has not been previously reported in simulations.

The periodicity of the shear-band formation appears to be less homogeneous for the deepest cut $(d=24$ $\mathrm{nm}$ ). For this cutting depth, the formation of a secondary shear band can be observed between the second and third shear band, see Fig. 2d. Secondary shear bands that grow between the serrations have also been observed in experiments [17]. Here, the secondary shear band grows parallel to the other shear bands without reaching the surface. In this particular case, we observe that the formation of the secondary shear band occurs after the formation of the third shear band, at the same instant when the fourth shear band starts to grow (see Fig. 5 below). This indicates a change in the cutting dynamics such that shear strain not necessarily only localizes along the primary shear zone (PSZ), see Fig. 1.

We identify the PSZ as the plane of the last shear band formed; it is marked in orange in Fig. 2. The PSZ also identifies the boundary of the chip. Since shear bands grow parallel to the PSZ, we use the coordinate perpendicular to the PSZ, $h$, to measure properties of the shear bands, and the coordinate parallel to the PSZ, $t$, to measure the chip width, $T$, as displayed in the inset of Fig. 1. 


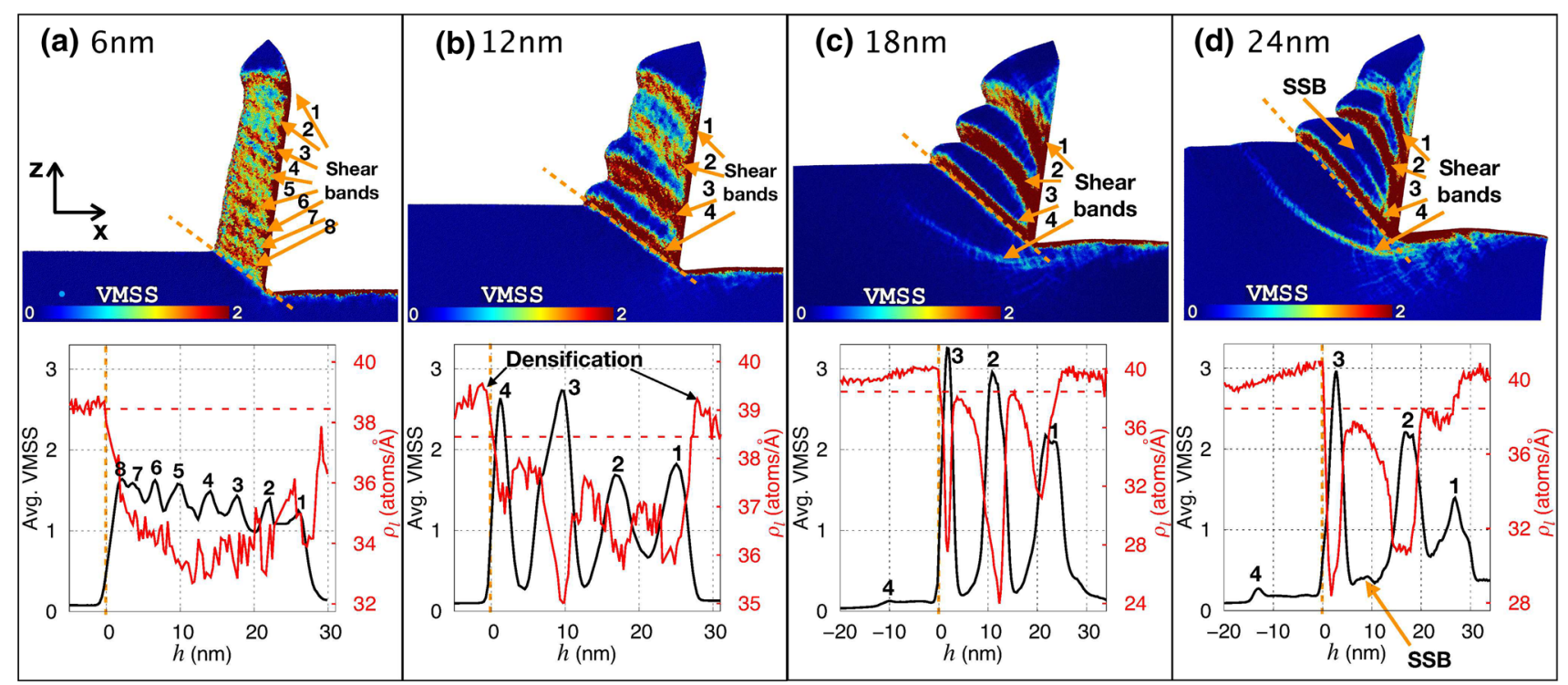

Fig. 2 Upper panel: VMSS snapshots at cutting length $l=40 \mathrm{~nm}$ for cutting depths a $6 \mathrm{~nm}, \mathbf{b} 12 \mathrm{~nm}, \mathbf{c} 18 \mathrm{~nm}$, and $\mathbf{d}$ $24 \mathrm{~nm}$. A front view in the $x-z$ plane is shown. Shear bands are numbered for easier reference; the appearance of a secondary shear band (SSB) in $\mathbf{d}$ is highlighted. The dashed orange line indicates the position of the PSZ (at $h=0)$ used to study the shear bands in the chip. Lower panel: average VMSS (black line) and atomic density, $\rho_{l}$, (red line) as a function of distance $h$ from the PSZ, see Fig. 1. Data are averaged over the $y$ direction perpendicular to the displayed sideview. The horizontal dashed red line denotes the average density before the cutting procedure. The orange dashed line at $h=0$ indicates the position of the PSZ shown in the upper panel

A quantitative analysis of the chip plasticity is displayed in the lower panel of Fig. 2. We focus on the variations of the VMSS and the atomic density within the chip, as these quantities are known to be affected at the core and in the vicinity of the shear bands $[28,50]$. To perform the analysis, we grid the chip into stripes parallel to the PSZ with a width of $2.77 \AA$; tool atoms are not included in this analysis. The graphs displayed in Fig. 2 show the averaged VMSS per stripe and the atom density in the stripe, $\rho_{l}$, defined as the number of atoms per stripe divided by the length of the stripe.

Figure 2 shows that in the shear bands, the VMSS increases, while the atomic density $\rho_{l}$ decreases. The increase in VMSS is the defining characteristic of a shear band as it quantifies shear localization. Also the decrease in $\rho_{l}$ in shear bands is well known [50] and is due to the atomic rearrangements occurring within a shear band. In Fig. 2, we compare the local-density values with the average before the deformation started.

For the deeper cuts, the VMSS in between shear bands is close to zero and the density is close to the initial average value of the undeformed sample. This demonstrates that the matrices of the shear bands do hardly interact with each other for cutting depths $d \geq 18 \mathrm{~nm}$. In addition, we consistently observe a slight density increase in the areas outside the shear bands, i.e., in the sample material below the chip and in the top part of the chip (see lower panel of Fig. 2b). These are caused by material compression during the shearband propagation.

In contrast, for the shallow cut, $d=6 \mathrm{~nm}$, Fig. 2a, the formed shear bands are so close together that the

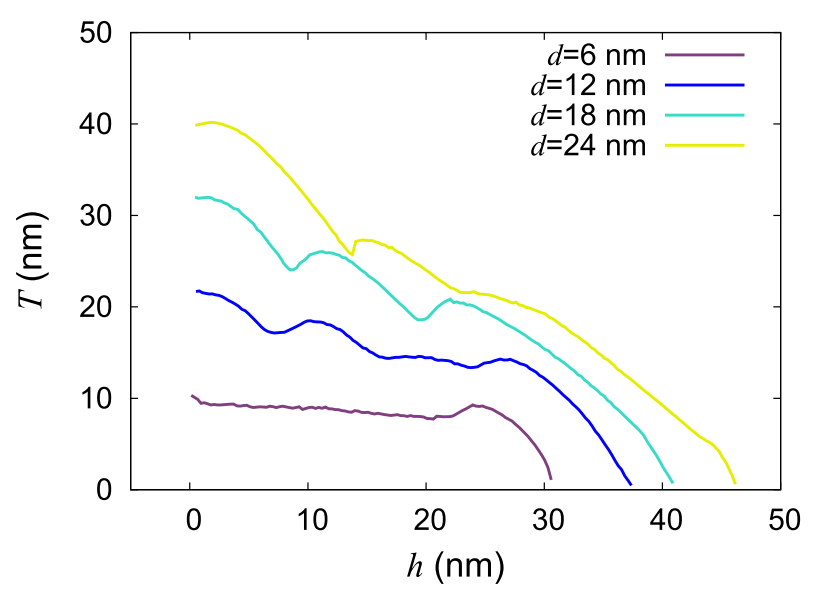

Fig. 3 Chip width, $T$, measured as a function of distance from the PSZ, $h$, see Fig. 1. Data are taken at the final cutting length $l=40 \mathrm{~nm}$ for all the simulated cutting depths $d$

contrast in VMSS between the core and the surrounding of the shear bands is small; this points to a considerable mutual interaction between shear bands such that individual shear bands could not evolve independently of each other. Simultaneously, the atomic density in the entire chip is strongly reduced with respect to the pristine sample, but no clear correspondence between shear bands and density minima is established. For this cutting depth, the density increase at the top and bottom of the chip is so small that it cannot be distinguished from fluctuations. This is similar to the findings in Ref. [9]. While larger density changes can be observed at the 
core and surroundings of the shear bands for deeper cuts, we feel uncertain to decide whether or not density changes play a direct role in the formation of the chip serrations.

The separation between shear bands, $\Delta_{\mathrm{SB}}$, increases with cutting depth. The corresponding values of $\Delta_{\mathrm{SB}}$ are assembled in Table 1. Most pronounced is the jump in $\Delta_{\mathrm{SB}}$ between cuts of $6 \mathrm{~nm}$ and $12 \mathrm{~nm}$ depth; this is indicative of a change in shear-band formation and will be analyzed further below, Sect. 3.2. Concomitant with the increase of shear-b'and spacing, the shear-band thickness $t_{\mathrm{SB}}$ grows, see Table 1 . While for the shallow $6-\mathrm{nm}$ cut, the shear-band thickness is only $1.5 \mathrm{~nm}$, it increases to roughly $4 \mathrm{~nm}$ for the deeper cuts. This indicates that with increasing depth, the strain amplitude also increases, which is expected since more material is being pushed by the cutting tool. In experiments, it has been observed that the width of the shear bands extends over 10-200 nm [27]. However, the scale of deformation in experiments is several orders of magnitude larger than in simulations. We thus expect a further increase of shear-band width when performing deeper cuts.

The chip profiles are displayed in Fig. 3, where the evolution of chip width, $T$, as a function of the distance to the PSZ, $h$, is shown. These data are calculated at the last stage of the simulation shown in the corresponding snapshots in Fig. 2. The figure demonstrates that both the chip thickness at the PSZ, $T_{\max }$, and the final chip length increase with cutting depth. The values of $T_{\max }$ are assembled in Table 1. Besides the chip serrations, which clearly show up in this plot for the deeper cuts, an overall trend is observed that chips become steadily thinner towards their upper ends; only for the shallowest cut, $d=6 \mathrm{~nm}$, the chip has a constant thickness and thus an approximately prismatic shape.

In previous simulations it has been shown that - for this particular metallic glass - the separation of the shear bands depends on further machining parameters such as the cutting velocity [6] and the rake angle of the tool [7]. As for the velocity dependence, Ref. [6] demonstrated that cutting the sample at a velocity of $10 \mathrm{~m} / \mathrm{s}$ results in thinner and less defined shear bands than the ones created at a cutting speed of $20 \mathrm{~m} / \mathrm{s}$. According to the findings in this study and in [6], we thus expect a similar transition in the growth behavior of the shear bands when cutting at slower speeds, but occurring for deeper cuts. This is of particular interest since experimental studies are performed at considerably slower velocities [11].

\subsection{Shear-band growth}

We study the propagation of shear bands by focusing on the third shear band as a representative example. The embryonic stage of this shear band, before full propagation, is shown for each of the cutting depths studied here in Fig. 4. The propagation direction of the third shear band for the shallowest cut $(d=6 \mathrm{~nm})$ differs from that for the deeper ones.
For the cutting depth of $6 \mathrm{~nm}$, the shear-band is initiated at the edge of the tool and then propagates straight towards the surface with an angle $\phi \sim 37^{\circ}$. However, for deeper cuts, the shear bands take a curved path. For cutting depths $\geq 12 \mathrm{~nm}$, the shear band initially emerges with a lower propagation angle - denoted by $\phi_{0}$ in Fig. 4 - from the tool edge. During propagation the angle increases until it eventually approaches the angle of the PSZ, $\phi$. These angles are tabulated in Table 1.

Further details of the time evolution of a shear band are provided in Fig. 5 for the deepest cut, $d=24 \mathrm{~nm}$. This series of snapshots starts after the stage displayed in Fig. 4d; the final state is seen in Fig. 2d. These snapshots show that considerable shear activity also spreads out into the sample beneath the cutting tool; the activity is in the form of isolated wave-like patterns that take the appearance of embryonic, not fully developing shear bands. The embryonic fourth shear band develops a curved trajectory, by propagating first towards the interior (Fig. 5b) and turning later towards the surface (Fig. 5c). Moreover, changes in the third shear band can be observed even after nucleation of the fourth shear band: the third shear band gets thicker and its curvature changes. This is an indication that the evolving shear strain keeps affecting this shear band.

We conclude that at shallow cutting depths, shear bands start from the tool edge and grow straight towards the surface. At larger depths, $d \gtrsim 12 \mathrm{~nm}$, shear bands exhibit a more complex growth behavior. Starting at a flat angle towards the cutting direction, or even in a direction towards the sample interior, they bend around towards the surface during continuing cutting. At this stage, shear bands are more distantly spaced from each other. Occasionally, incipient (secondary) shear bands are produced, but they stop developing before reaching the surface, without maturing into full-fledged bands.

\section{Discussion}

Laminar plastic flow, Fig. 6a, is a simple paradigm for cutting. The assumption of mass conservation above the flow stagnation point at the tool edge leads to a prismatic chip geometry ('straight chip') and simple predictions about the chip width $d^{\prime}: d^{\prime}=d \cos (\phi-\alpha) / \sin \phi$ $[1,2]$. We recently demonstrated [7] that this model well describes the results of atomistic simulations of metallic-glass cutting (cutting depth $d=7 \mathrm{~nm}$ ) for a large range of rake angles $\alpha$.

In the present study, we see large deviations from this model occurring at larger cutting depths, as sketched schematically in Fig. 6b. At first sight, this appears astonishing, since a metallic glass is a homogeneous medium that has no intrinsic length scale besides the atom sizes, which are too small to be of relevance in cutting. Thus from the point of view of continuum mechanics, all cutting results for different depth should scale on each other after normalizing to the cutting depth; in particular, chip shapes should be prismatic and similar 


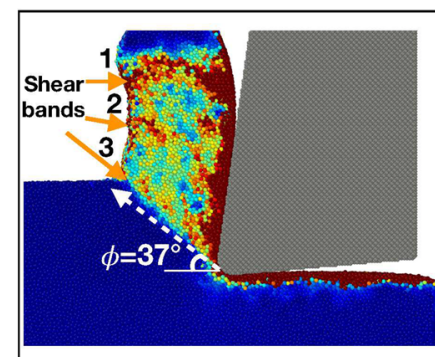

(a) $6 \mathrm{~nm}$

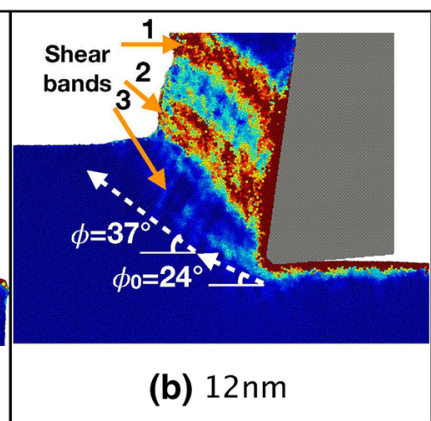

(b) $12 \mathrm{~nm}$
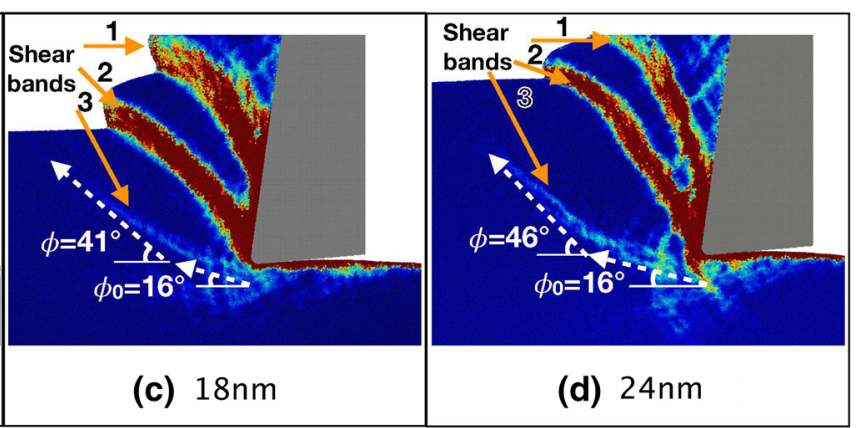

Fig. 4 VMSS snapshots showing the initial growth of the third shear band for cutting depths a $6 \mathrm{~nm}$ (cutting length $l=14.6 \mathrm{~nm}), \mathbf{b} 12 \mathrm{~nm}(l=20.6 \mathrm{~nm}), \mathbf{c} 18 \mathrm{~nm}(l=28.8 \mathrm{~nm})$, and $\mathbf{d} 24 \mathrm{~nm}(l=21.4 \mathrm{~nm})$. The white arrows highlight the direction of the shear-band propagation. The propagation mode changes from an initial angle $\phi_{0}$ towards the final angle $\phi$ in the case of the deeper cuts $(\mathbf{b}-\mathbf{d})$

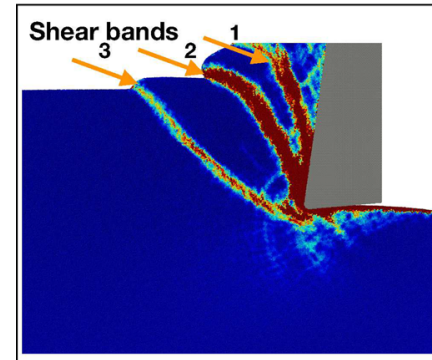

(a) $l=27 \mathrm{~nm}$

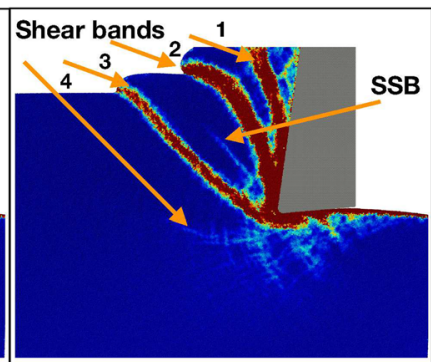

(b) $l=31 \mathrm{~nm}$
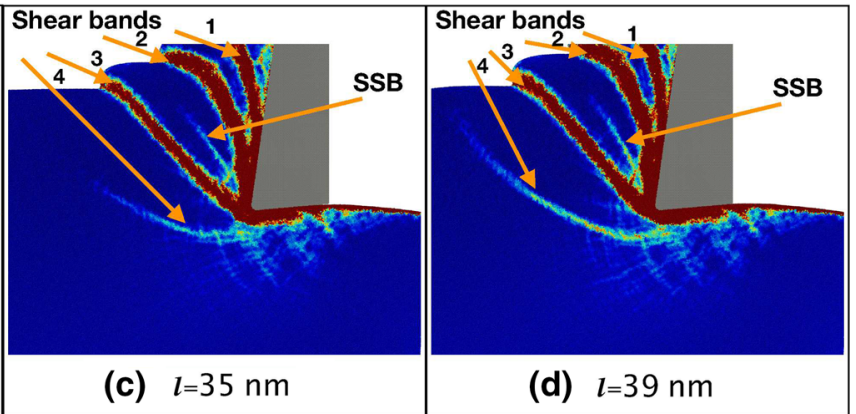

Fig. 5 VMSS snapshots showing the growth of the third shear band for the deepest cut $(d=24 \mathrm{~nm})$ for cutting lengths of a $27 \mathrm{~nm}, \mathbf{b} 31 \mathrm{~nm}$, c $35 \mathrm{~nm}$, and $\mathbf{d} 39 \mathrm{~nm}$. Shear bands are numbered and the appearance of a secondary shear band (SSB) is highlighted

Table 1 Chip characteristics for the cutting depths $d$ studied: average shear-band separation, $\Delta_{\mathrm{SB}}$; average shear-band thickness, $t_{\mathrm{SB}}$; chip thickness at the PSZ, $T_{\max }$; initial angle of shear-band propagation, $\phi_{0} ;$ angle of the PSZ, $\phi$

\begin{tabular}{llllll}
\hline Depth $(\mathrm{nm})$ & $\Delta_{\mathrm{SB}}(\mathrm{nm})$ & $t_{\mathrm{SB}}(\mathrm{nm})$ & $T_{\max }(\mathrm{nm})$ & $\phi_{0}$ & $\phi$ \\
\hline 6 & 3.9 & 1.5 & 9.36 & - & $37^{\circ}$ \\
12 & 8.0 & 3.9 & 21.74 & $24^{\circ}$ & $37^{\circ}$ \\
18 & 10.4 & 4.2 & 32.52 & $16^{\circ}$ & $41^{\circ}$ \\
24 & 12.0 & 4.2 & 41.01 & $16^{\circ}$ & $46^{\circ}$ \\
\hline
\end{tabular}

in the geometric sense of the word. This is clearly not the case for our simulation results.

As outlined in the Introduction, in metallic glasses, plasticity is governed by shear bands which introduce their own length scales. To be concrete, the shearband spacing, $\Delta_{\mathrm{SB}}$, serves as an additional length scale. While the shear-band spacing is tiny $(3.9 \mathrm{~nm})$ for the 6 $\mathrm{nm}$ cut such that shear bands almost merge, for deeper cuts, a finite number of shear bands is created with a spacing $\Delta_{\mathrm{SB}}$ of around $10 \mathrm{~nm}$. The occurrence of these shear bands may be interpreted as an instability caused by strain localization by shear bands.

Instabilities in plastic flow have been observed previously in the cutting of crystalline metals. Scraping a nanocrystalline $\mathrm{Cu}$ sample with a hard tool gave rise to a serrated chip surface, which was attributed to a so-called folding instability of the plastic flow
[51]. The experiments were accompanied by molecular dynamics simulations which demonstrated that folding is caused by the plastic inhomogeneity of the nanocrystalline material. Figs. 1e and $1 \mathrm{f}$ in that reference show that the grain boundaries and grain interiors suffer different strains - quite analogous to our Fig. 2 where shear bands and the material in between show different shears. Macroscopic experiments of metal cutting exhibited a so-called sinuous flow accompanied by mushroom-like surface structures and wavy surface patterns on the $100-\mu \mathrm{m}$ scale $[52,53]$. The resulting chips were characterized by a sawtooth morphology.

Also in sliding contacts of multilayered material with layer thicknesses of $100 \mathrm{~nm}$, a surface instability towards an undulation and 'vortex formation' was reported [54]. Sliding of polycrystalline material was 

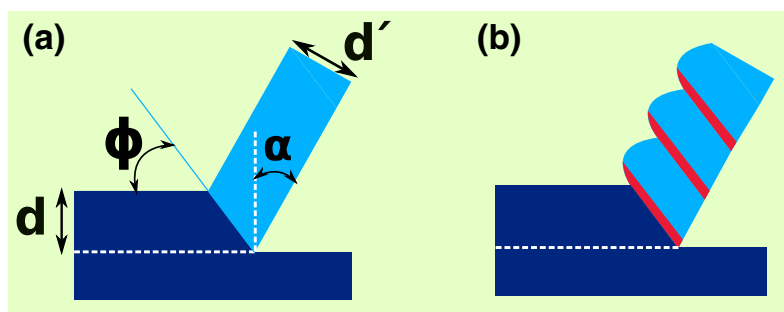

Fig. 6 Schematics showing the difference between chip formation in a laminar flow leading to a straight chip and $\mathbf{b}$ unstable flow leading to a serrated chip. Important cutting characteristics of the cutting process are included in a: depth of cut $d$; width of straight chip, $d^{\prime}$; rake angle, $\alpha$; angle between PSZ and cutting direction, $\phi$

found in simulations to lead to grain coarsening and rotation caused by shear localization [55].

Earlier sliding experiments of large-grained (grain size $118 \mu \mathrm{m}) \mathrm{Cu}$ surfaces [56,57] showed non-laminar plastic flow giving rise to bump and fold formation upstream of the contact zone. This behavior was interpreted to be akin to Kelvin-Helmholtz instabilities in hydrodynamic flows; this analogy was however argued to be accidental by Beckmann et al. [51].

We conclude that, while previous simulations of metallic-glass cutting have not shown chip serration, our results are in line with molecular-dynamics simulations of machining nanocrystalline metals and with experiments on polycrystalline metals. The common origin of serration lies in all cases in the instability of the laminar plastic flow caused by strain localization. In the case of metallic glasses, this localization is due to shear bands, while grain boundaries and other defects play this role in nano-crystalline material.

\section{Conclusion}

Cutting of metallic glasses leads for shallow cuts to straight prismatic chips such as they should be expected for an isotropic material assuming laminar plastic flow $[1,2]$. For deeper cuts, however, the peculiar mechanism of plasticity of metallic glasses - viz. strain localization in shear bands - interferes by disturbing the laminar plastic flow to produce serrated chips. This mechanism sets in as soon as shear bands are sufficiently separated that they do not interact with each other; in our study that occurs for a shear-band separation of around 10 $\mathrm{nm}$ which is manifested at cutting depths beyond 12 $\mathrm{nm}$. The waviness of the chip surface then reproduces the shear-band separation.

In atomistic detail, we could demonstrate that for these deeper cuts, there is a change of the shear-band propagation mechanism. While for shallower cuts the shear bands propagate along the PSZ with a fixed angle, for deeper cuts the initial propagation angle shifts to a larger angle before reaching the surface. This more complex shear-band propagation mechanism is responsible for the increasing shear-band separation at deeper cuts and thus for the plastic-flow instability.

We acknowledge support by the Deutsche Forschungsgemeinschaft (DFG, German Research Foundation) - Project Number 172116086 - SFB 926 and IRTG 2057 - Project Number 252408385. Fruitful discussions with Stefan Küchemann are acknowledged. Simulations were performed at the High Performance Cluster Elwetritsch (RHRK, TU Kaiserslautern, Germany).

\section{Author contributions}

$\mathrm{KA}$ and VV performed the simulations and analyzed the results. KA, VV and HMU designed the work and discussed the results. KA and HMU wrote the manuscript.

Funding Open Access funding enabled and organized by Projekt DEAL.

Data Availability Statement This manuscript has no associated data or the data will not be deposited. [Authors' comment: There are no new data associated with this paper other than those generated in the simulation runs.]

Open Access This article is licensed under a Creative Commons Attribution 4.0 International License, which permits use, sharing, adaptation, distribution and reproduction in any medium or format, as long as you give appropriate credit to the original author(s) and the source, provide a link to the Creative Commons licence, and indicate if changes were made. The images or other third party material in this article are included in the article's Creative Commons licence, unless indicated otherwise in a credit line to the material. If material is not included in the article's Creative Commons licence and your intended use is not permitted by statutory regulation or exceeds the permitted use, you will need to obtain permission directly from the copyright holder. To view a copy of this licence, visit http://creativecomm ons.org/licenses/by/4.0/.

\section{References}

1. M.E. Merchant, J. Appl. Phys. 16, 267 (1945a)

2. M.E. Merchant, J. Appl. Phys. 16, 318 (1945b)

3. P.L.B. Oxley, Int. J. Mech. Sci. 3, 68 (1961)

4. A.G. Atkins, Int. J. Mech. Sci. 45, 373 (2003)

5. A.G. Atkins, Eng. Fract. Mech. 72, 849 (2005)

6. K.E. Avila, S. Küchemann, I. Alabd Alhafez, H.M. Urbassek, J. Appl. Phys. 127, 115101 (2020a)

7. K.E. Avila, V.H. Vardanyan, I. Alabd Alhafez, M. Zimmermann, B. Kirsch, H.M. Urbassek, J. Non-Cryst. Solids 550, 120363 (2020b)

8. V.H. Vardanyan, K.E. Avila, S. Küchemann, H.M. Urbassek, Comput. Mater. Sci. 192, 110379 (2021)

9. K.E. Avila, S. Küchemann, H.M. Urbassek, J. NonCryst. Solids 566, 120882 (2021) 
10. K. Fujita, Y. Morishita, N. Nishiyama, H. Kimura, A. Inoue, Mater. Trans. 46, 2856 (2005)

11. M. Bakkal, A.J. Shih, R.O. Scattergood, C.T. Liu, Script Mater. 50, 583 (2004)

12. M. Bakkal, V. Naksiler, Mater. Manuf. Processes 24, $1249(2009)$

13. M.Q. Jiang, L.H. Dai, Acta Mater. 57, 2730 (2009)

14. F. Zeng, M.Q. Jiang, L.H. Dai, Proc. R. Soc. A: Math. Phys. Eng. Sci. 474, 20170836 (2018)

15. X. Chen, J. Xiao, Y. Zhu, R. Tian, X. Shu, J. Xu, Mater. Des. 136, 1 (2017)

16. L. Zhang, H. Huang, Int. J. Adv. Manuf. Technol. 100, 637 (2019)

17. F. Ding, C. Wang, T. Zhang, L. Zheng, X. Zhu, W. Li, L. Li, J. Mater. Process. Technol. 276, 116404 (2020)

18. R. Maaß, J.F. Löffler, Adv. Funct. Mater. 25, 2353 (2015)

19. A.L. Greer, Y.Q. Cheng, E. Ma, Mater. Sci. Eng. R Rep. 74, $71(2013)$

20. Y. Zhang, A.L. Greer, Appl. Phys. Lett. 89, 071907 (2006)

21. T.C. Hufnagel, C.A. Schuh, M.L. Falk, Acta Mater. 109, 375 (2016)

22. J.-O. Krisponeit, S. Pitikaris, K.E. Avila, S. Küchemann, A. Krüger, K. Samwer, Nat. Commun. 5, 3616 (2014)

23. C. Liu, A. Das, W. Wang, S. Küchemann, P. Kenesei, R. Maaß, Scripta Mater. 170, 29 (2019)

24. R. Maaß, P. Birckigt, C. Borchers, K. Samwer, C.A. Volkert, Acta Mater. 98, 94 (2015)

25. H.S. Shahabi, S. Scudino, I. Kaban, M. Stoica, B. Escher, S. Menzel, G.B.M. Vaughan, U. Kühn, J. Eckert, Acta Mater. 111, 187 (2016)

26. S. Küchemann, C. Liu, E.M. Dufresne, J. Shin, R. Maaß, Phys. Rev. B 97, 014204 (2018)

27. C. Liu, V. Roddatis, P. Kenesei, R. Maaß, Acta Mater. 140, 206 (2017)

28. A.J. Cao, Y.Q. Cheng, E. Ma, Acta Mater. 57, 5146 (2009)

29. G. Subhash, H. Zhang, Metall. Mater. Trans. A 38, 2936 (2007)

30. S. Scudino, D. Şopu, Nano Lett. 18, 1221 (2018)

31. D. Şopu, A. Stukowski, M. Stoica, S. Scudino, Phys. Rev. Lett. 119, 195503 (2017)

32. Y. Shi, M.L. Falk, Acta Mater. 55, 4317 (2007)

33. K.E. Avila, S. Küchemann, I. Alabd Alhafez, H.M. Urbassek, Materials 12, 1477 (2019a)

34. K. E. Avila, S. Küchemann, I. Alabd Alhafez, H. M. Urbassek, Tribol. Int. 139, 1 (2019b)

35. K.E. Avila, S. Küchemann, H.M. Urbassek, J. NonCryst. Solids 523, 119593 (2019c)
36. C. Zhong, H. Zhang, Q.P. Cao, X.D. Wang, D.X. Zhang, U. Ramamurty, J.Z. Jiang, J. Non-Cryst. Solids 445446, 61 (2016)

37. C.-D. Wu, Appl. Phys. A 122, 486 (2016)

38. Y. Zhao, X.L. Wei, Y. Zhang, F.H. Wu, D.H. Huo, Key Eng. Mater. 667, 88 (2015)

39. Y. Zhao, T. Li, Y. Zhang, D. Huo, Curr. Nanosci. 13, 48 (2016)

40. C.-D. Wu, T.-H. Fang, J.-K. Su, Appl. Surf. Sci. 396, 319 (2017)

41. S. Plimpton, J. Comput. Phys. 117, 1 (1995). http:// lammps.sandia.gov/

42. M.I. Mendelev, D.J. Sordelet, M.J. Kramer, J. Appl. Phys. 102, 043501 (2007)

43. Y.-C. Wang, C.-Y. Wu, J.P. Chu, P.K. Liaw, Metall. Mater. Trans. A 41, 3010 (2010)

44. C. J. Ruestes, E. M. Bringa, Y. Gao, H. M. Urbassek, in Applied Nanoindentation in Advanced Materials, edited by A. Tiwari and S. Natarajan (Wiley, Chichester, UK, 2017), chap. 14, pp. 313-345

45. B. Luan, M.O. Robbins, Phys. Rev. E 74, 026111 (2006)

46. R. Narulkar, S. Bukkapatnam, L.M. Raff, R. Komanduri, Comput. Mater. Sci. 45, 358 (2009)

47. P. Stoyanov, P.A. Romero, R. Merz, M. Kopnarski, M. Stricker, P. Stemmer, M. Dienwiebel, M. Moseler, Acta Mater. 67, 395 (2014)

48. S. Goel, G. Cross, A. Stukowski, E. Gamsjäger, B. Beake, A. Agrawal, Comput. Mater. Sci. 152, 196 (2018)

49. A. Stukowski, Model. Simul. Mater. Sci. Eng. 18, 015012 (2010). http://www.ovito.org/

50. A.D.S. Parmar, S. Kumar, S. Sastry, Phys. Rev. X 9, 021018 (2019)

51. N. Beckmann, P.A. Romero, D. Linsler, M. Dienwiebel, U. Stolz, M. Moseler, P. Gumbsch, Phys. Rev. Appl. 2, 064004 (2014)

52. H. Yeung, K. Viswanathan, W.D. Compton, S. Chandrasekar, Proc. Natl. Acad. Sci. USA 112, 9828 (2015)

53. H. Yeung, K. Viswanathan, A. Udupa, A. Mahato, S. Chandrasekar, Phys. Rev. Appl. 8, 054044 (2017)

54. Z.-P. Luo, G.-P. Zhang, R. Schwaiger, Script. Mater. 107, 67 (2015)

55. P.A. Romero, T.T. Järvi, N. Beckmann, M. Mrovec, M. Moseler, Phys. Rev. Lett. 113, 036101 (2014)

56. N.K. Sundaram, Y. Guo, S. Chandrasekar, Phys. Rev. Lett. 109, 106001 (2012)

57. S. de Beer, M.H. Müser, Physics 5, 100 (2012) 